\title{
Prevalence of Missing First Permanent Molars in a Selected Population in a University Dental Clinic Setting: A Retrospective Radiographic Study
}

\author{
Yousef M Almugla
}

\begin{abstract}
Aim and objective: This study aimed to determine the prevalence of missing first permanent molars (FPMs) in the 7-29-year-old selected population of Al Ahsa, Saudi Arabia.

Materials and methods: This study retrospectively analyzed 398 panoramic radiographs of patients aged from 7 to 29 years old for missing FPM. Patients were divided into two age-groups: 7-15 and 16-29 years old. Study variables including gender, age, missing FPM, dental arch, and right or left sides of the arch were recorded. The data were statistically analyzed using Chi-square tests.

Results: Of the total study sample, $23.1 \%$ of patients had only one missing FPM, $13.3 \%$ of patients had two, and $2.8 \%$ of them had three missing FPM teeth. No patient had four missing FPM teeth. A total of $60.8 \%$ (242) radiographs in the overall sample did not show any missing teeth. The mandibular FPMs (35.2\%) were found missing more frequently than their maxillary counterparts (17.4\%) and the left mandibular quadrant showed a higher rate of missing FPM (21.9\%). No statistically significant association was seen between the missing FPM and the gender or age-group $(p>0.05)$.

Conclusion: The high prevalence rates of missing FPM in the study population warrants attention. Further prospective studies are needed to comprehensively evaluate the effect of early loss of the FPM on space changes in the arch and subsequent reduction in complex orthodontic treatment needs.
\end{abstract}

Keywords: Age, Early loss, Missing tooth, Permanent first molar, Prevalence.

International Journal of Clinical Pediatric Dentistry (2021): 10.5005/jp-journals-10005-1941

\section{INTRODUCTION}

Tooth loss continues to be considered a major public health problem affecting the quality of life of an individual. ${ }^{1}$ There has been an increasing trend in the prevalence of dental caries and periodontal disease in Saudi Arabia in recent decades, which has been attributed to the increased consumption of sugary foods and carbonated drinks and lack of knowledge regarding proper oral health care. ${ }^{2,3}$ First permanent molars (FPMs) are among the earliest erupting permanent teeth in the oral cavity and have been observed as most caries-prone teeth in the mixed dentition. ${ }^{4}$ In addition, these teeth are more vulnerable to hypomineralization and periodontal diseases ${ }^{5}$ and may result in early tooth loss due to unmet treatment needs. Moreover, FPMs play a crucial part in the development of occlusion and arch integrity, and early loss of these teeth may affect spatial relation, which could significantly affect the symmetry of dentition. ${ }^{6}$

The quantification of missing FPM in a population might be the first step toward understanding the trends and characteristics of the severity of malocclusion and therefore the complexity of orthodontic treatment needs. Panoramic radiographs are most commonly used by orthodontists as a diagnostic record and play an important role in examination, diagnosis, and orthodontic treatment planning. Studies estimating the rate of missing or an early loss of FPM among the population seeking orthodontic treatment are scarce in Saudi Arabia, especially in the Eastern province region. Hence, this study aimed to determine the prevalence of missing FPM teeth in 7-29-year-old patients using panoramic radiographic images.
Faculty in Orthodontics, Department of Preventive Dental Sciences, College of Dentistry, King Faisal University, Al Ahsa, Eastern Province, Kingdom of Saudi Arabia

Corresponding Author: Yousef M Almugla, Faculty in Orthodontics, Department of Preventive Dental Sciences, College of Dentistry, King Faisal University, Al Ahsa, Eastern Province, Kingdom of Saudi Arabia, e-mail: yalmugla@kfu.edu.sa; yalmugla@gmail.com

How to cite this article: Almugla YM. Prevalence of Missing First Permanent Molars in a Selected Population in a University Dental Clinic Setting: A Retrospective Radiographic Study. Int J Clin Pediatr Dent 2021;14(2):269-272.

Source of support: Nil

Conflict of interest: None

\section{Materials and Methods}

The present retrospective study included 398 panoramic radiographs of patients, randomly selected from the digital archives of the dental clinics complex of the King Faisal University. These patients had reported seeking various dental treatment procedures during the period 2018 to 2020 . The panoramic images used in this study were previously acquired for diagnostic purposes. Panoramic radiographs of only those patients aged 7-29 years showing an optimal viewing and diagnostic quality were included in this study. Radiographs with blurred images or severe distortion resulting in unreliable diagnosis were excluded. Written informed consent had been taken from all patients as part of a routine protocol for the use of collected data solely for research and educational purposes. 
The study was approved by the Institutional Research and Ethics Committee (KFU-REC/2020-11-07) and carried out in accordance with the Declaration of Helsinki.

All the panoramic radiographs were acquired by an experienced oral radiology technician, using an i-CAT tomography unit (Imaging Sciences International, Hatfield, PA, USA). These images were taken at $120 \mathrm{kVp}, 2-15 \mathrm{~mA}$ at different resolutions; with an exposure time of 2-8 seconds and a voxel of $0.3 \mathrm{~mm}$. Demographic information including gender, age, and the reason for missing FPM was obtained from the patient's records. Missing FPM teeth in both the maxillary and mandibular arches on the right or left side of the arch were determined from panoramic radiographs. Teeth indicated for extraction were not recorded.

One well-trained and experienced examiner (YMA) evaluated all the panoramic radiographs and recorded the data. The intra-examiner reliability was calculated using an unweighted $\mathrm{k}$ coefficient. Statistical analyses were performed using the Statistical Package for the Social Sciences (SPSS), version 20.0 (SPSS Inc., Chicago, IL, USA). Data were analyzed using descriptive statistics to obtain the absolute and percentage frequency. Chi-square tests were applied to verify the existence of significant associations among the variables. The significance level was set at $p<0.05$.

\section{Results}

The present study assessed panoramic radiographs of 398 patients, including 190 males (47.7\%) and 208 females (52.3\%), aged between 7 years and 29 years. The subjects were divided into two groups based on age and eruption status of the second permanent molar, i.e., group I (7-15 years) and group II (16-29 years). The assessment of intra-examiner reproducibility revealed а к of 0.97 .

The overall prevalence of missing FPM in the present study was $39.2 \%$. Of the total study sample, $92(23.1 \%)$ patients had only one missing FPM, 53 (13.3\%) patients had two, and 11 (2.8\%) of them had three missing FPM teeth. No patient had four missing FPM teeth. A total of $60.8 \%$ (242) radiographs in the overall sample did not show any missing teeth. The distribution of missing FPM by the age-group and gender of the subjects is summarized in Table 1. No statistically significant association was seen between the gender and missing FPM (Chi-square value: $2.359 ; p=0.124$ ).

In the 7- to 15-year-old group, 61 (25.3\%) subjects had one missing FPM followed by 22 (9.1\%) with two missing, and 6 (2.5\%) with three missing FPM. On the other hand, the 16-29-year-old group showed that 31 (19.7\%) of them had one missing FPM, 31 (19.7\%) with two missing, and 5 (3.2\%) of them with three missing FPM, respectively (Table 1 ). There was no statistically significant association between the study age-groups and missing FPM (Chi-square value: $1.317 ; p=0.251$ ). According to the distribution of missing FPM relative to the dental arch, the mandibular FPMs (35.2\%) were found missing more frequently than their maxillary counterparts (17.4\%). The left mandibular quadrant showed the highest number of missing FPM (21.9\%). The distribution of missing FPM teeth according to the jaw or dental arch is shown in Table 2. No significant association was noted between gender and dental arches with regard to missing FPM (Chi-square value: $0.029 ; p=$ 0.865).

Missing mandibular FPMs were seen in 140 (35.2\%) of the subjects. Of these, 120 (30.2\%) subjects had one missing and 20 (5\%) of them had two missing mandibular FPM. The right mandibular FPM was missing in 76 (19.1\%) subjects and the left mandibular FPM was missing in 87 (21.9\%). Maxillary FPMs were found missing in 69 (17.4\%) subjects. Among these, 64 (16.1\%) had one and 05 (1.3\%) subjects had two missing maxillary FPM. In the maxillary arch, 36 (9\%) of the subjects presented missing FPMs in each of the right and left sides (Table 3). The Chi-square test showed that the association between the number of missing FPM on the right or left sides of the dental arch was not significant (Chi-square value: $0.731 ; p=0.392$ ).

\section{Discussion}

The present retrospective study investigated the prevalence of missing FPM using panoramic radiographs of 7-29-year-old subjects and found that about $39.2 \%$ of the study population had at least one missing FPM. The age-group studied here constitutes the orthodontic population as most people who seek orthodontic treatment are seen within the age-group selected in the present study. This study focused exclusively on the FPM since they are the first teeth to erupt into the oral cavity and are more prone to caries. Moreover, FPMs are considered keystones of dentition and have a crucial role in the development of occlusion. These teeth are considered to be the best source of anchorage for orthodontic tooth movement as they have maximum root surface area. ${ }^{7}$ As FPMs play an important role in arch integrity and masticatory function, especially among those aged $<14$ years, early loss of these teeth may affect the occlusion significantly in these group of patients. ${ }^{8}$

Table 2: Frequency of teeth missing according to the dental arch

\begin{tabular}{lcc}
\hline & Maxilla & Mandible \\
\hline One missing & $64(16.1 \%)$ & $120(30.2 \%)$ \\
Two missing & $05(1.3 \%)$ & $20(5 \%)$ \\
No missing & $329(82.7 \%)$ & $258(64.8 \%)$ \\
\hline
\end{tabular}

Table 3: Frequency of missing FPM in all four quadrants

\begin{tabular}{lllll}
\hline & Missing 16 & Missing 26 & Missing 36 & Missing 46 \\
\hline Male & $13(6.8 \%)$ & $17(8.9 \%)$ & $29(15.3 \%)$ & $37(19.5 \%)$ \\
Female & $23(11.1 \%)$ & $19(9.1 \%)$ & $58(27.9 \%)$ & $39(18.8 \%)$ \\
Total & $36(9 \%)$ & $36(9 \%)$ & $87(21.9 \%)$ & $76(19.1 \%)$ \\
$p$ value & $p=0.143^{*}$ & $p=0.948$ & $p=0.004^{*}$ & $p=0.854$ \\
\hline
\end{tabular}

*Statistically significant

Table 1: Frequency of teeth missing according to age-group and gender

\begin{tabular}{lllllll}
\hline Gender & One missing $n(\%)$ & Two missing $n(\%)$ & Three missing $n(\%)$ & No missing $n(\%)$ & Total $n(\%)$ & $p$ value \\
\hline Male & $43(22.6 \%)$ & $20(10.5 \%)$ & $04(2.1 \%)$ & $123(64.7 \%)$ & 190 & 0.124 \\
Female & $49(23.6 \%)$ & $33(15.9 \%)$ & $07(3.4 \%)$ & $119(57.2 \%)$ & 208 & \\
Total & $92(23.1 \%)$ & $53(13.3 \%)$ & $11(2.8 \%)$ & $242(60.8 \%)$ & 398 & \\
Age (years) & & & & & & \\
$7-15$ & $61(25.3 \%)$ & $22(9.1 \%)$ & $06(2.5 \%)$ & $152(63.1 \%)$ & $241(100 \%)$ & 0.251 \\
$16-29$ & $31(19.7 \%)$ & $31(19.7 \%)$ & $05(3.2 \%)$ & $90(57.3 \%)$ & $157(100 \%)$ & \\
\hline
\end{tabular}


While the early loss of FPM at a chronological age of 8-10 years facilitates mesial movement of the second permanent molar into the FPM area, ${ }^{9}$ loss of FPM at later stages, i.e., during or after the eruption of the second permanent molar may result in inadequate space closure. ${ }^{10}$ Raducanu et al..$^{11}$ in their study reported that the loss of FPM after the age of 11 years does not result in spontaneous space closure. They found a significant association between the age at which FPM was lost and the post-extraction space. However, in the present retrospective study, it was not possible to precisely determine when the loss of the FPM occurred.

Previous studies have reported prevalence rates of missing FPM in the range of $8-57 \%$ and had included subjects within 7-20 years of age. ${ }^{12,13}$ Similarly, in the present study, the prevalence of missing FPM in the 7-15-year age-group was $36.9 \%$ while it was $42.7 \%$ in the 16-29-year age-group. The difference between agegroups with regard to missing FPM was not significant $(p=0.251)$. This study found that about $25.3 \%$ of subjects aged $7-15$ years had one missing FPM, 9.1\% had two missing molars, and $2.5 \%$ presented three missing molars. Rezaie et al. ${ }^{14}$ in a study on the Iranian population found that $24 \%$ of children aged $7-15$ years had one missing FPM followed by $31 \%$ with two missing molars and $15 \%$ with three missing molars. Differences in findings between other studies might be due to variations in study design, age, and population groups.

Multiple studies in the literature have reported that the lower FPMs were found missing more frequently than the upper FPM. ${ }^{15,16}$ Halicioglu et al. ${ }^{10}$ in a study on $13-20$-year-old Turkish group found that lower FPMs were more frequently missing (66\%) than the upper FPMs (34\%) and the difference was statistically significant. Bhat et al. ${ }^{17}$ determined the prevalence of missing mandibular and maxillary FPMs to be $21 \%$ and $10 \%$, respectively. However, Demirbuga et al. ${ }^{18}$ had reported lower rates of missing permanent first molar teeth in the mandible $(2.77 \%)$ and maxilla $(1.17 \%)$ compared with other studies. In this study, the most frequently missing tooth type was the first lower permanent molar, which was consistent with the literature. The lower FPMs demonstrated a significantly higher missing rate (35.2\%) than their maxillary counterparts (17.4\%). Further, the lower-left FPM ( $p=$ 0.002 ) showed statistically significant higher missing rates in the females than the other FPM (Table 3). This finding corroborates with previous studies in Saudi Arabia wherein a significantly higher DMFT (decayed, missing, and filled teeth) in mandibular molars were reported than in maxillary molars. However, it should be noted that the present study focused only on missing FPM. In addition, this study included subjects in the 7-29-year age-group, while previous studies had explored populations with a wider age range. Several factors have been related to the higher rate of caries involvement of the mandibular FPM compared with their maxillary counterparts such as the morphology, earlier eruption times of lower FPM, and greater plaque accumulation in the mandibular posterior region. ${ }^{14,19}$

Studies exploring the reasons for missing teeth found that dental caries and periodontal disease are the main reasons for tooth mortality, with the former being the more prevalent reason among subjects younger than 40 years of age and the latter in those aged 40 years and above. ${ }^{4,15}$ Studies conducted on the Saudi population reported that caries was the most common cause of tooth loss, which corroborates with the present study. ${ }^{13,20}$ Consistent with the literature, this study found that caries was the dominant reason for early loss of all the FPM (100\%), and there was no loss of teeth due to periodontal disease or other reasons.

The influence of gender on tooth loss has been reported in the literature with a female predilection to higher tooth loss rates. ${ }^{18,21}$ Halicioglu et al. ${ }^{10}$ in a study on 13-20-year-old Turkish group found that the gender difference was not significant with regard to missing FPM. Other authors had affirmed that tooth loss was affected more by the age factor than by gender. ${ }^{22}$ This study displayed that $42.8 \%$ of females and $35.3 \%$ of males had at least one missing FPM, and the association between gender and missing FPM was not significant $(p=0.124)$. Studies have found no significant difference between missing FPM on the right or left side of the dental arches. ${ }^{17,18}$ Similar to previous studies, this study found no statistically significant difference between the number of missing FPM on the right or left side of the arches $(p=0.392)$. However, lower left FPM was found to be missing more frequently than the FPM in other quadrants, which corroborates with the findings of other authors. ${ }^{14}$

The present study is the first in a series of investigations analyzing the prevalence of missing FPM in 7-29-year-old patients who attended the University dental clinical complex. In the present study, while only missing FPMs were studied, the high prevalence rates of these teeth in the age-group studied usually representing the orthodontic population pose serious concern regarding increased malocclusion severity thereby affecting the complexity and the duration of orthodontic treatment causing financial burden. While Saudi Arabia provides free public orthodontic treatment services, individuals with severe treatment needs face delayed access to relevant facilities compared with those with minor orthodontic treatment procedures. ${ }^{23}$ Therefore, it is necessary to increase awareness of the importance of FPM and the consequences caused by the early loss of FPM teeth.

One limitation in the present study was that this study had small sample size and was conducted on patients who sought dental care at the dental clinical complex of King Faisal University, Al Ahsa in the Eastern province of Saudi Arabia, and hence, one can expect that these individuals present with more dental treatment needs than does the general population. Therefore, generalization of results must be made with caution as this study population may not reflect the prevalence of early loss of FPM teeth among the similar population in other regions of Saudi Arabia.

\section{Conclusion}

The results of this study indicate that the prevalence of missing FPM in the sample population was high (39.2\%) and mandibular FPMs were more frequently missing than maxillary teeth. The lower left FPM was the most frequently missing tooth (21.9\%). The high prevalence rates of FPM tooth loss in the study population warrants attention regarding the promotion of educational awareness and provision of dental care to preserve these teeth and/or otherwise provide timely orthodontic intervention to reduce the complexity of associated sequelae. Further prospective studies are needed to comprehensively evaluate the spatial relationship affected by the early loss of the FPM and subsequent reduction in complex orthodontic treatment needs.

\section{ACKnOWLedgments}

The study was approved by the Research and Ethics Committee of King Faisal University (KFU-REC/2020-11-07). The author is grateful 
to the Deanship of Scientific Research, King Faisal University, Al Ahsa, for facilitating this study.

\section{REFERENCES}

1. Gerritsen AE, Allen PF, Witter DJ, et al. Tooth loss and oral healthrelated quality of life: a systematic review and meta-analysis. Health Qual Life Outcomes 2010;8(1):126. DOI: 10.1186/1477-7525-8-126.

2. Al-Ansari AA. Prevalence, severity, and secular trends of dental caries among various Saudi populations: a literature review. Saudi J Med Med Sci 2014;2(3):142-150. DOI: 10.4103/1658-631X.142496.

3. Farooqi FA, Khabeer A, Moheet IA, et al. Prevalence of dental caries in primary and permanent teeth and its relation with tooth brushing habits among schoolchildren in Eastern Saudi Arabia. Saudi Med J 2015;36(6):737-742. DOI: 10.15537/smj.2015.6.10888.

4. Ali D. Reasons for extraction of permanent teeth in a university dental clinic setting. Clin Cosmet Investig Dent 2021;13:51-57. DOI: 10.2147/ CCIDE.S294796.

5. Jafarian M, Etebarian A. Reasons for extraction of permanent teeth in general dental practices in Tehran, Iran. Med Princ Pract 2013;22(3):239-244. DOI: 10.1159/000345979.

6. Caglaroglu M, Kilic N, Erdem A. Effects of early unilateral first molar extraction on skeletal asymmetry. Am J Orthod Dentofacial Orthop 2008;134(2):270-275. DOI: 10.1016/j.ajodo.2006.07.036.

7. Arrow P. Dental enamel defects, caries experience and oral healthrelated quality of life: a cohort study. Aust Dent J 2017;62(2):165-172. DOI: 10.1111/adj.12449.

8. Jalevik B, Moller M. Evaluation of spontaneous space closure and development of permanent dentition after extraction of hypomineralized permanent first molars. Int J Paediatr Dent 2007;17(5):328-335. DOI: 10.1111/j.1365-263X.2007.00849.x.

9. Gill DS, Lee RT, Tredwin CJ. Treatment planning for the loss of first permanent molars. Dent Update 2001;28(6):304-308. DOI: 10.12968/ denu.2001.28.6.304.

10. Halicioglu K, Celikoglu M, Caglaroglu M, et al. Effects of early bilateral mandibular first molar extraction on condylar and ramal vertical asymmetry. Clin Oral Investi 2013;17(6):1557-1561. DOI: 10.1007/ s00784-012-0843-9.

11. Raducanu AM, Feraru V, Herteliu C, et al. Prevalence of loss of permanent first molars in a group of Romanian children and adolescents. Oral Health Dent Manag 2009;8(3):3-10.
12. Ozmen B. Evaluation of permanent first molar tooth loss in young population from north Turkey. Balk J Dent Med 2019;23(1):20-23. DOI: 10.2478/bjdm-2019-0004.

13. Atieh MA. Tooth loss among Saudi adolescents: social and behavioural risk factors. Int Dent J 2008;58(2):103-108. DOI: 10.1111/j.1875595x.2008.tb00184.x.

14. Rezaie M, Ghapanchi J, Haghnegahdar A, et al. A radiographic evaluation of missing of permanent first molars in a group of Iranian children and adults: a retrospective study. Int J Dent 2018;2018:5253965. DOI: 10.1155/2018/5253965.

15. Al-Shammari KF, Al-Ansari JM, Al-Melh MA, et al. Reasons for tooth extraction in Kuwait. Med Princ Pract 2006;15(6):417-422. DOI: 10.1159/000095486.

16. Artun J, Thalib L. Mesial migration and loss of first molars among young adolescents in Kuwait. Community Dent Health 2011;28(2):154-159.

17. Bhat N, Mitra R, Reddy JJ, et al. Reasons and pattern of tooth mortality as perceived by dental professionals in Udaipur city, Rajasthan, India. Arch Oral Res 2013;9(2):149-157. DOI: 10.7213/ archivesoforalresearch.09.002.AO01.

18. Demirbuga S, Tuncay O, Cantekin K, et al. Frequency and distribution of early tooth loss and endodontic treatment needs of permanent first molars in a Turkish pediatric population. Eur J Dent 2013;7(Suppl 1):S099-S104. DOI: 10.4103/1305-7456.119085.

19. Passarelli PC, Pagnoni S, Piccirillo GB, et al. Reasons for tooth extractions and related risk factors in adult patients: a cohort study. Int J Environ Res Public Health 2020;17(7):2575. DOI: 10.3390/ ijerph17072575.

20. Alesia K, Khalil HS. Reasons for and patterns relating to the extraction of permanent teeth in a subset of the Saudi population. Clin Cosmet Investig Dent 2013;5:51-56. DOI: 10.2147/CCIDE.S49403.

21. George B, John J, Saravanan S, et al. Prevalence of permanent tooth loss among children and adults in a suburban area of Chennai. Indian J Dent Res 2011;22(2):364. DOI: 10.4103/0970-9290.84284.

22. Susin C, Haas AN, Opermann RV, et al. Tooth loss in a young population from south Brazil. J Public Health Dent 2006;66(2):110-115. DOI: 10.1111/j.1752-7325.2006.tb02565.x.

23. Alajlan SS, Alsaleh MK, Alshammari AF, et al. The prevalence of malocclusion and orthodontic treatment need of school children in Northern Saudi Arabia. J Orthod Sci 2019;8(1):10. DOI: 10.4103/jos. JOS_104_18. 